\title{
Malignant peripheral nerve sheath tumour (malignant epithelioid Schwannoma) of the parotid gland
}

\author{
De Stefano $A^{1}$, Kulamarva $\mathrm{G}^{2}$, Citraro $\mathrm{L}^{1}$, Borgia $\mathrm{L}^{3}$, Croce $\mathrm{A}^{1}$ \\ Dipartimento di Scienze Chirurgiche, Sperimentali e Cliniche: Istituto di Otorinolaringoiatria Università degli Studi \\ "G. d'Annunzio” Chieti-Pescara, Italia.dr.adestefano@gmail.com
}

\begin{abstract}
Problems/objectives: Malignant peripheral nerve sheath tumours are uncommon soft tissue tumours originating from Schwann cells or nerve sheath cells. Malignant epithelioid schwannoma is an aggressive variant of malignant peripheral nerve sheath tumour, and unfortunately is related with a high rate of recurrence and poor prognosis.

Methodology: In this study we present a rare case of malignant epithelioid schwannoma of the parotid gland and we discuss its origin, unusual presentation, and possible treatments options.

Results: Patient underwent total parotidectomy without neck dissection. Postoperative radiotherapy and chemotherapy management were not necessary.

Conclusion: Patients with malignant epithelioid schwannomas typically present with pain and/or rapid enlargement of a pre-existing lesion but the diagnosis of these tumours remains difficult as it is based primarily on clinical suspicion. There is no recognized management for this tumour yet while the prognosis seems to correlate best with the extent of surgical resection (Fig. 4, Ref. 16). Full Text in PDF www.elis.sk.

Key words: malignant schwannoma, parotid gland, malignant peripheral nerve sheath tumors, parotidectomy, S-100 protein, vimentin.
\end{abstract}

Malignant peripheral nerve sheath tumours (MPNSTs) are uncommon soft tissue tumours originating from Schwann cells or nerve sheath cells (1). World Health Organization coined the term MPNST to identify any malignant tumour arising from a peripheral nerve.

Most MPNSTs involve the proximal portions of extremities and a few occur in the head and neck regions. The latter pattern is a characteristic that contrasts with normal distribution of benign schwannomas (1).

Malignant epithelioid schwannoma is an aggressive variant of MPNST and unfortunately is related with a high rate of recurrence and poor prognosis (2). In extracranial nerve branches, MES presents with symptoms such as pain or weakness $(2,3,4)$. These tumours typically follow a malignant course with local recurrence and haematogenous metastases despite surgical resection.

There is no recognized optimal management for this tumour yet while the prognosis seems to correlate best with the extent of surgical resection (5).

This current report is on a rare case of MES of the parotid gland without facial nerve palsy. In the article we also discuss its origin, unusual presentation, and possible treatments options.

${ }^{1}$ Dipartimento di Scienze Chirurgiche, Sperimentali e Cliniche: Istituto di Otorinolaringoiatria Università degli Studi "G. d'Annunzio" Chieti-Pescara, Italia, ${ }^{2}$ Ent \& Head- Neck Department: Thekkil, Complex Kasaragod, Kerala State, India, and ${ }^{3}$ Istituto di Anatomia Patologica e Citodiagnostica, Università degli Studi “G. d'Annunzio" Chieti-Pescara, Chieti, Italia Address for correspondence: A. De Stefano, MD, Via di Palma 10, 74100-Taranto, Italy.

Phone:+393475111044

\section{Case report}

A 64-year-old male presented to us seeking attention for a right-sided parotid mass with associated hemifacial pain. At presentation, he had been suffering from this condition for one year. There was no past history of Bell's palsy, facial weakness or facial paraesthesia. He gave no history of previous radiation and the family history was non-contributory to patient's condition.

Clinical examination revealed a hard mass in the right parotid region extending inferiorly towards the neck. It was fixed to the underlying muscles, tender to touch, and covered with healthy skin.

There was no evidence of cervical lymphadenopathy. Facial nerve function was normal. Patient underwent a computed tomography $(\mathrm{CT})$ scan of the parotid region and neck which revealed a nodular high-density mass of $2.5 \mathrm{x} 4 \mathrm{~cm}$ in the right superficial parotid gland. The deep lobe or parapharyngeal space was not involved by the mass. There were no calcified or cystic areas seen in the affected parotid gland (Fig. 1).

Bilateral renal insufficiency and medical health problems prevented the patient from undergoing MRI examination of the tumour. Two successive fine-needle aspiration cytologies did not give us the definitive diagnosis. In view of this a superficial parotidectomy was planned.

Unfortunately during the surgery, the tumour was found to be involving the superficial and deep lobes of parotid gland (Fig. 2). Frozen biopsy was performed and it was suggestive of malignant nervous tumour. The greyish, lobulated, hard mass was totally removed. It appeared not to be arising from the parotid tissue. Being not involved by the tumour, the facial nerve with its principal 


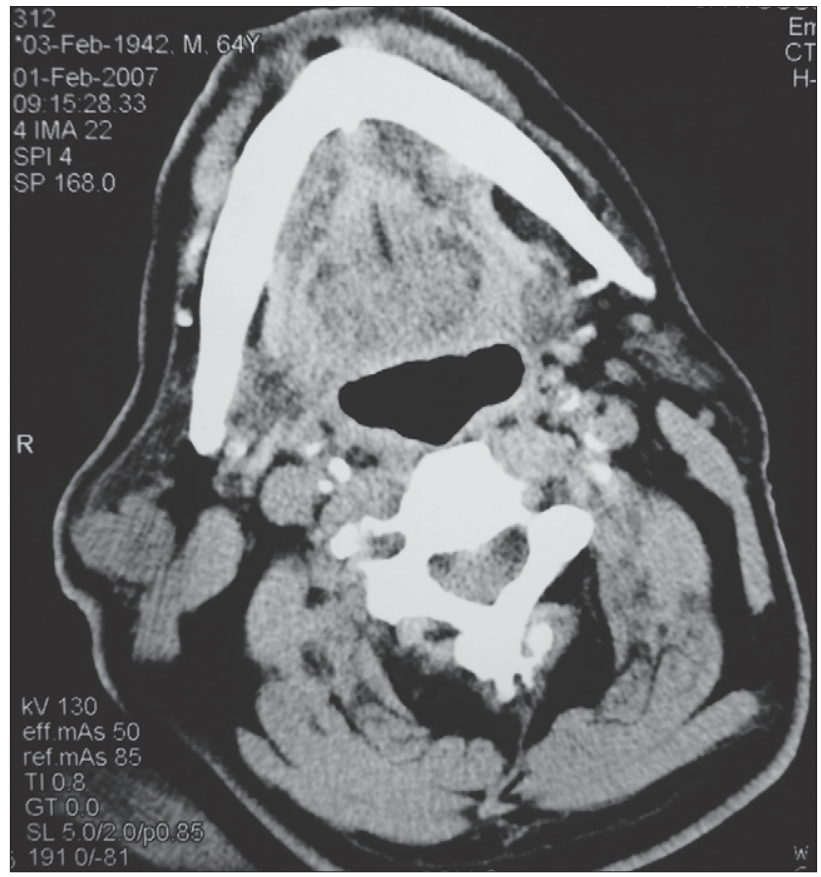

Fig. 1. Axial CT scan of head and neck showed a nodular high-density mass of $2.5 \times 4 \mathrm{~cm}$ in diameter in right superficial parotid gland. The deep lobe and the right parapharyngeal space were not involved by the mass. There were no calcified or cystic areas seen in the affected parotid gland.

branches were identified and preserved. The surgical procedure was completed by removing the deep lobe. It was not deemed necessary to dissect the lymph node. The margins around the resected tumour were clear from disease.

Histological evaluation of the tumour showed nests of epithelioid cells admixed with spindle-shaped components. The cords of schwann cells with a pleomorphic epithelioid appearance were arranged in a vague nodular pattern (Fig. 3). The cells had large round nuclei with prominent melanoma-like nucleoli. Based on these features, the tumour was identified as an epithelioid malignant schwannoma.

The tumour had densely cellular areas with a high mitotic index as well as necrotic areas. Groups of cells were separated by myxoid stroma depending on the accumulation of acid mucin. Subtle blending of the epithelioid areas with spindle areas was often observed in the tumour.

Immunoistochemical examination confirmed the diagnosis. The tumour was strongly and diffusely positive for S-100 protein and vimentin. Lack of immunoreactivity for cytokeratins and Melan- A/ MART1 and HMB-45 by the tumour cells excluded the diagnosis of synovial sarcoma or melanoma (Fig. 4). No adjuvant treatment protocol (radiation or chemotherapy) was followed after the surgery. The patient is free of disease 12 months after the surgery.

\section{Discussion}

Malignant epithelioid schwannoma is a rare, poorly differentiated spindle cell sarcoma originating from the supportive non-

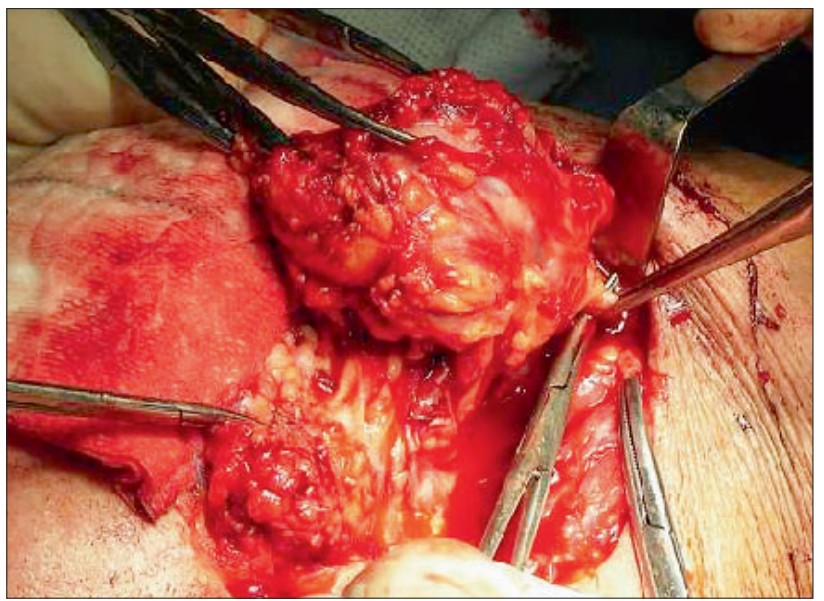

Fig. 2. Intraoperative photograph showing the tumour arising from the superficial lobe of parotid gland.

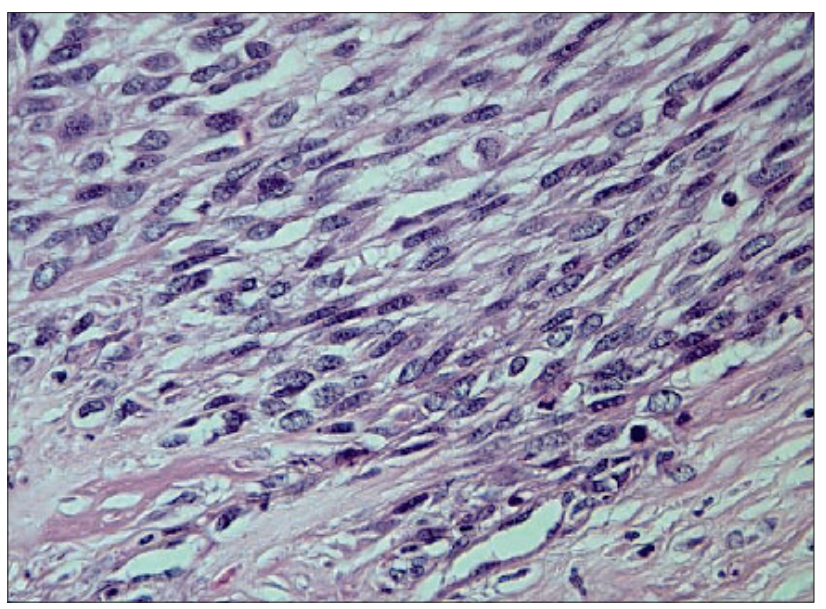

Fig. 3. Histological evaluation of the tumour showed nests of epithelioid cells admixed with spindle shaped components. Cords of schwann cells with a pleomorphic epithelioid appearance were arranged in a vague nodular pattern.

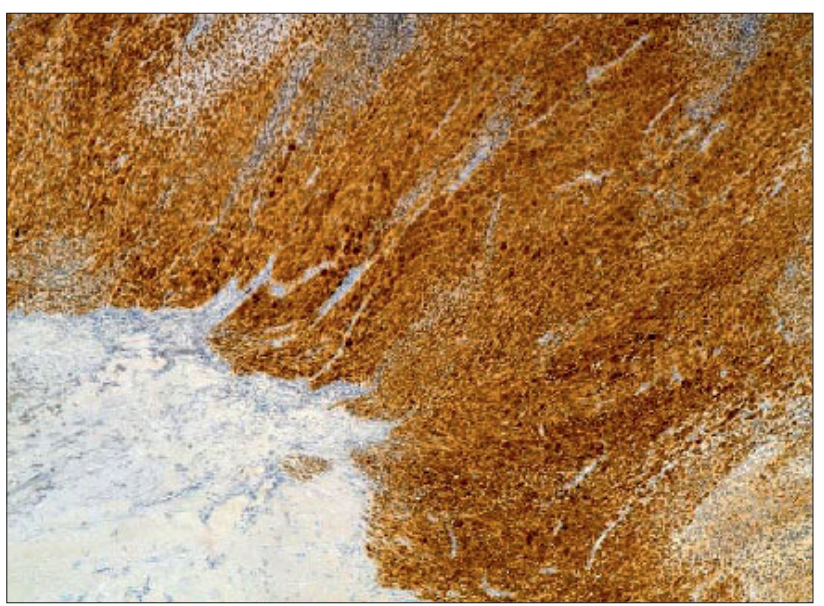

Fig. 4. Immunohistochemistry evaluation: tumour was strongly and diffusely positive for S-100 protein and Vimentin. 
neuronal components of peripheral nerves (7). These tumours typically arise from malignant transformation of a pre-existing benign peripheral nerve sheath tumour such as a neurofibroma (8).

Benign peripheral nerve sheath tumours rarely undergo malignant change. In those rare instances where this occurs, the tumour takes the form of either MPNST, or more exceptionally, that of angiosarcoma $(9,10)$. In 1994, Woodruff et al. reviewed nine cases of MPNST arising from benign schwannomas. It was notable that in seven of the nine cases, the malignant tumour was purely epithelioid in morphology (8). Malignant epithelioid schwannoma is estimated to comprise only $5 \%$ of all cases of MPNSTs. This variant of sarcoma is not usually associated with the neurofibromatosis type 1 (9). These tumours infiltrate widely and diffusely into the surrounding tissues, and also readily metastasise through the bloodstream to the chest, liver, bone and brain (11). Spread through lymphatic vessels is very uncommon, thereby casting some doubts regarding the utility of neck dissection (11) in such cases. In our present case we chose to perform total right parotidectomy with facial nerve preservation without neck dissection. This was because intraoperatively, the facial nerve with all its principal branches was normal and the tumour did not appear to be arising from the nerve itself such as seen in a facial nerve schwannoma. Also, pre-operative computed tomography scans of head and neck and intraoperative analysis of lymph nodes were negative for metastases.

\section{Diagnosis}

The diagnosis of these tumours remains difficult as it is based primarily on clinical suspicion. Patients with malignant extracranial schwannomas typically present with pain and/or rapid enlargement of a pre-existing lesion. Particularly the pain seems to be a more reliable symptom of malignancy in these cases (1).

In accordance with other published reports in English literature, our patient too experienced a mass in his right parotid gland with associated significant hemifacial pain. The preoperative diagnosis of parotid MESs is very difficult because MPNSTs of the major salivary glands are extremely rare. They constitute $0.3 \%$ to $1.5 \%$ of all salivary gland tumours (12). Clinically most MPNSTs of salivary glands are localized in the parotid, probably because of great quantity of neural structures in this gland (11). These tumours can often be asymptomatic. There is no gender or racial predilection, and the symptoms (except for pain) are not characteristics. Only $10 \%$ of patients develop facial nerve palsy (11).

Radiologic detection and evaluation of soft-tissue tumours have become increasingly important with the advent of CT and MR imaging. Magnetic resonance imaging (MRI) is the current gold standard in MPNSTs imaging. The information on enhancing qualities of the mass obtained from MRI combined with its appearance on T1- and T2-weighted images can give valuable clues as to its histopathology (13). Areas of haemorrhage, necrosis, heterogeneous enhancement and cystic areas may suggest malignancy but are by no means definitive and can occasionally be seen in benign neoplasms too.

Computed tomography (CT) appears to be of limited value in diagnosing these lesions. However, CT provides a unique ability to demonstrate the relation of a soft-tissue mass to its surrounding structures including muscles, bone and neurovascular bundles (14). In our case, CT scan revealed a nodular high-density mass in the right superficial parotid gland. There were no calcifications or cystic areas seen in the right parotid gland. Our patient's medical health problems prevented him from undergoing MRI scan evaluation.

Given that the resection of tumour with clear margins requires sacrifice of vital nerve and soft tissue structures, some surgeons advocate fine-needle aspiration (FNA) before surgery in MPNSTs management. FNA of malignant epithelioid schwannoma shows aggregations of spindle cells and occasional detached single cells. Pleomorphism is variable and related to grading of the sarcoma. The cytoplasm is typically indistinct and naked nuclei are common while Dodd revealed that this variant of sarcoma may not be seen characteristically on FNA examination (15). In our present case report, two consecutive FNAs were in fact negative for cytological diagnosis.

\section{Treatment}

Once the diagnosis of MPNST is suspected, surgical treatment is the best choice. The aim of surgery is to remove the tumour completely while achieving tumour-free margins (13). Definitive surgical treatment should show clear tumour margins for at least two centimetres on all sides. (13).

Lymphatic neck dissection is not necessary in these patients (11). The reported local recurrence rate of MES following gross total resection is $32 \%$ to $65 \%$ after intervals of 5 to 32 months (11). Locally aggressive resection is thought to decrease the risk of systemic metastasis (13).

We treated our patient with total right parotidectomy while preserving the facial nerve. In fact, intra-operatively, the superficial and deep lobes of the parotid gland were involved by the tumour. The mass was excised completely along with adequate margins. The facial nerve was not involved by the lesion and therefore was preserved. In fact when the nerve is functioning normally preoperatively, it should be preserved during surgery whenever possible, and in these cases, the oncological prognosis does not deteriorate.

No neck dissection was necessary and no chemotherapy or radiotherapy was administered postoperatively.

In fact, as exposed by the European Organization for Research and Treatment of Cancer, chemotherapy is not curative (16). Although adjuvant high-dose therapy has little effect on long term survival rates, it can be used particularly after incomplete resection or when total resection of the tumour is unattainable.

\section{Histological evaluation and immunohistochemistry}

The epithelioid variant of MPNST is typically composed of round-to-polygonal cells arranged in nodules or nests with high mitotic index, as well as of necrotic areas. Individual tumour cells are plump, spindle cells with round and/or serpentine nuclei. Nuclear pleomorphism and hyperchromatism are consistent features (2). As described in literature, histological evaluation of our case showed fusiform spindled cells with round nuclei and prominent melanoma-like nucleoli. Immunohistochemistry plays an impor- 
tant role in the histological evaluation. Immunoreactivity to Vimentin and S-100 (like in our patient; Fig 4) and negative staining for markers of epithelial, neuroendocrine or muscular differentiation are hallmarks of MPNST (11).

\section{Conclusion}

Malignant epithelioid schwannoma is the most aggressive variant of MPNST and it is rarely related to neurofibromatosis. It usually arises from malignant change in a benign schwannoma. Major salivary glands are rarely involved by these sarcomas but when this happens, the parotid gland is the principal location.

Diagnosis of MESs is very difficult because patients can be asymptomatic or may complain only of pain or tenderness. Only up to $10 \%$ may have facial palsy or weakness. The fact that pathological and radiological differential diagnoses are closely intermingled entails the necessity of a careful multidisciplinary approach.

Surgical management is the best choice and a radical tumour excision is recommended with facial nerve preservation when it is functionally normal and free of disease.

Chemotherapy is usually not indicated and adjuvant radiotherapy should be given only when the disease is incompletely excised. Unfortunately even with the best treatment the patient is burdened with a high recurrence rate and poor prognosis.

\section{References}

1. Enzinger FM, Weiss SW. Soft-tissue tumors. St Louis: Mosby, 3rd edition 1995: 889-910.

2. De Rubeis DA, Woulfe J, Rosso D, Lownie S, Parnes L, Lee D, Strong MJ, Hammond RR. Case of the month: June 1997: a 42 year old man with left facial weakness. Brain Pathol 1997; 8: 233-234.

3. Muhlbauer MS, Clark WC, Robertson JH, Gardner LG, Dohan FC. Malignant nerve sheath tumor of the facial nerve: case report, discussion. Neurosurgery 1987; 21: 68-73.

4. Habal MB: Malignant epithelioid schwannoma of the mandible and the skull base. J Craniofacial Surg 1997; 8: 417-421.
5. McGuirt 3 WF, Johnson PE, McGuirt WT. Intraparotid facial nerve neufibromas. Laryngoscope 2003;113: 82-84.

6. Hamakawa H, Kayahara H, Sumida T, Tanioka H. Mandibular malignant schwannoma with multiple spinal metastases: a case report and review of the literature. J Oral Maxillofac Surg 1998; 56: 1191-1196.

7. Matsuda Y, Saoo K, Hosokawa K, Yamakawa K, Yokohira M, Zeng Y, Takeuchi H, Iwai J, Shirai T, Obika K, Imaida K. Epithelioid malignant peripheral nerve sheath tumor. Report of a case with inflammatory infiltration. Pathol Res Pract 2005; 201: 355-360.

8. Woodruff JM, Selig AM, Crowley K, Allen PW. Shwannoma (Neurilemona) with malignanttransformation. Am J Surg Pathol 1994; 18 : 882-895.

9. Di Carlo EF, Woodruff JM, Bansal M et al. The purely epithelioid malignant peripheral nerve sheath tumor. Am J Surg Pathol 1986; 10: 478-490.

10. McMenamin ME, Fletcher CD. Expanding the spectrum of malignant change in schwannomas: epithelioid malignant change, epithelioid malignant peripheral nerve sheath tumor, and epithelioid angiosarcoma: a study of 17 cases. Am J Surg Pathol 2001; 25: 13-25.

11. Punjabi AP, Haug RH, Chung-Park MJ, Likavek M. Malignant peripheral nerve sheath tumor of the parotid gland: report of case. J Oral Maxillofac Surg 1996; 54: 765-769.

12. Luna MA, Tortoledo ME, Ordóñez NG, Frankenthaler RA, Batsakis JG. Primary sarcomas of the major salivary glands. Arch Otolaryngol Head Neck Surg 1991; 117: 302-306.

13. Gupta G, Maniker A. Malignant peripheral nerve sheath tumors. Neurosurg Focus 2007; 22: 1-8.

14. Kransdorf MJ. Malignant Soft-Tissue Tumors in a Large Referral Population: Distribution of Diagnoses by Age, Sex, and Location. AJR 1995; 164: 129-134.

15. Dodd LG, Scully S, Layfield LJ. Fine-needle Aspiration of epithelioid malignant peripheral nerve sheath tumor (Epithelioid Malignant Schwannoma). Diagn Cytopathol 1996; 17: 200-204.

16. Santoro A, Tursz T, Mouridsen H, Verweyij J, Steward W, Sommers $\mathbf{R}$ et al. Doxorubicin versus CYVADIC versus Doxorubicin plus Ifosfamide in first line treatment advanced soft tissue sarcomas: a randomized study of the European Organization for Reserch and treatment of Cancer soft tissue and bones. Sarcoma group. J Clin Oncol 1995; 13: 1537-154.

Received July 6, 2010. Accepted June 26, 2012. 